\title{
Photovoltaic and optical properties of a polymer-PbS nanocomposite
}

\author{
P. Lutsyk ${ }^{1}$, L. Dzura ${ }^{2}$, A. Kutsenko ${ }^{3}$, Ya. Vertsimakha ${ }^{1}$, J. Sworakowski ${ }^{4}$ \\ ${ }^{I}$ Institute of Physics, NAS of Ukraine, 46, prospect Nauky, 03028 Kyiv, Ukraine \\ ${ }^{2}$ Taras Shevchenko Kyiv National University, Physics Department, \\ 6, prospect Academician Glushkov, 02033 Kyiv, Ukraine \\ ${ }^{3}$ Institute of Physical Chemistry, NAS of Ukraine, 31, prospect Nauky, 03039 Kyiv, Ukraine \\ ${ }^{4}$ Wroclaw University of Technology, Institute of Physical and Theoretical Chemistry, \\ Wyb. Wyspianskiego 27, 50-370 Wroclaw, Poland
}

\begin{abstract}
Photovoltaic and optical properties of a polymer-PbS nanoparticles in polyvinyl alcohol (PVA) films and the effect of annealing in air at various temperatures on these properties are studied in this work. In the range of 1.0 to $3.7 \mathrm{eV}$, absorption spectra of $\mathrm{PbS}$ nanocomposite films are correctly fitted by five Gaussian bands peaking at $1.5,1.84,2.41,3.06$, and $3.58 \mathrm{eV}$. A characteristic feature of the films is the presence of green and blue luminescence with intensity maxima at 2.25 and $3.02 \mathrm{eV}$, respectively. The luminescence intensity strongly depends on the excitation energy and the annealing temperature in air. The highest intensity of the green luminescence is observed under the excitation with $2.35 \mathrm{eV}$ radiation, whereas the intensity of the blue luminescence peaks under $3.46 \mathrm{eV}$ excitation. After annealing at $370 \mathrm{~K}$, the intensity of the green luminescence decreases a few times whereas the intensity of the blue emission increases. The photovoltaic sensitivity of the $\mathrm{PbS}$ nanocomposite films depends on the annealing conditions, attaining the maximum after annealing in air at approximately glass transition temperature of PVA $(370 \mathrm{~K})$. The spectral range of the photosensitivity of $\mathrm{PbS}$ nanocomposite films is wider than that of $\mathrm{CdSe}$ - and $\mathrm{CuInS}_{2}$-polymer nanocomposite films.
\end{abstract}

Keywords: $\mathrm{PbS}$ nanocomposite, absorption spectra, luminescence, photovoltage.

Manuscript received 26.04.05; accepted for publication 25.10.05.

\section{Introduction}

Nanocomposites of organic and inorganic semiconductors dispersed in polymer matrices are multifunctional materials often suggested as elements of nonlinear optical devices $[1,2]$ and for development of plastic solar cells $[3,4]$. The increasing interest in their possible use is caused by their plasticity, simple preparation techniques, and an opportunity to tune their properties by changing the concentration and the nanoparticle size in the matrix.

A polymeric nanocomposite should possess high optical and photochemical stability. Therefore, we have used polyvinyl alcohol (PVA) polymeric matrix and inorganic semiconductor $\mathrm{PbS}$ for nanocomposite preparation. PVA is practically transparent for the visible and near UV light, and $\mathrm{PbS}$ is chemically stable. Additionally, the small bandgap energy of $\mathrm{PbS}$ single crystals should allow to change absorption and photosensitivity of the nanocomposite over a wide spectral range by changing the concentration of the nanoparticles and their sizes. Finally, our choice was motivated by an important third order nonlinear optical efficiency, previously described in the PbS-PVA nanocomposite [5].

The primary goal of this work was a complex research of optical and photovoltaic properties of polymer-PbS nanocomposite films. We also set our mind on examining a possibility of fabrication of nanostructures photosensitive over a wide spectral range.

\section{Experimental}

The nanocomposite films have been prepared by spinning colloidal water solutions of $\mathrm{PbS}$ nanoparticles (19 wt.\%) stabilized by PVA on glass substrates. Two kinds of substrates were used: plain glass was employed to fabricate samples for optical measurements, whereas substrates covered with conducting indium tin oxide (ITO) were used as transparent electrodes in 
photovoltaic experiments. $\mathrm{PbS}$ nanoparticles have been obtained mixing aqueous solutions of $\mathrm{Pb}\left(\mathrm{NO}_{3}\right)_{2}$ and $\mathrm{Na}_{2} \mathrm{~S}$, followed by dialysis to remove $\mathrm{Na}^{+}$and $\mathrm{NO}_{3}{ }^{-}$ ions. Technical details of obtaining the composite films have been described in [5].

The size of $\mathrm{PbS}$ particles embedded into the polymer matrix was determined from the broadening the X-ray diffraction peaks. The X-ray diffraction pattern is shown in Fig. 1. Four peaks appearing in the pattern with the maxima at $2 \theta=26.1^{\circ}, 30.2^{\circ}, 43.1^{\circ}$ and $50.6^{\circ}$, were identified due to $\mathrm{PbS}$, their positions coinciding with those of reflexes determined for bulk PbS crystals. The fifth peak appearing at ca $20^{\circ}$ was attributed to PVA.

The average size of particles $D$ was determined from Sherrer's equation [6]:

$D=\frac{K \cdot \lambda}{\Delta \cos \theta}$,

where $K$ is the geometrical factor taken equal to 1 for the crystal structure of $\mathrm{PbS}$ (NaCl-type lattice), $\lambda$ is the $\mathrm{X}$ ray wavelength $(\lambda=1.542 \AA), \theta-$ stands for the scattering angle, and $\Delta=\left(B^{2}-B_{s}{ }^{2}\right)^{0.5}$ is a measure of the peak broadening. The parameter $B$ is the width of a peak in the X-ray diffraction pattern (in units of $2 \theta$ ), and $B_{s}$ is the calibration factor determined from the peak broadening of Si nanoparticles $\left(B_{s}=0.38^{\circ}\right)$. From the Xray pattern shown in Fig. 1, we have estimated the average size of $\mathrm{PbS}$ nanoparticles $D=(5 \pm 1) \mathrm{nm}$.

For the X-ray analysis we have used the DRON-4.07 diffractometer $\left(\mathrm{Cu} K_{\alpha}\right.$ radiation). Absorption spectra were measured by the spectrophotometer Unicam UV-300, spectral dependence of luminescence and its excitation - with "Hitachi" spectrophotometer.

The photovoltaic properties of the composite have been measured in the Bergman method modified by I. Akimov [7]. The method does not require any upper ohmic contact on the samples which is an important advantage as the technology of deposition of such

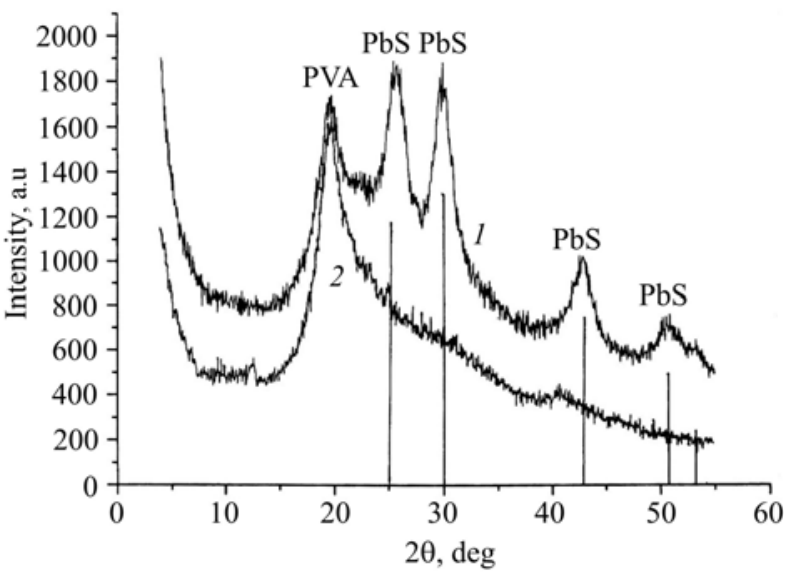

Fig. 1. X-ray diffraction pattern of a polymer-PbS nanocomposite in the PVA matrix (1) and PVA film (2). The vertical lines show the positions of reflexes in bulk PbS crystals. contacts has not been mastered yet. During the measurements, the samples were illuminated with modulated light of different wavelengths $(80 \mathrm{~Hz}, 1.3$ to $2.7 \mathrm{eV}$ ) from light-emitting diodes (LEDs). The photovoltage was measured with a Unipan type 232B nanovoltmeter. The modulated radiation power of LEDs was measured with a calibrated pyroreceiver and the silicon photodiode.

\section{Results and discussion}

\subsection{Absorption}

In the range of 1.3 to $4.2 \mathrm{eV}$, the absorption spectrum of a colloidal solution of $\mathrm{PbS}$ nanoparticles stabilized by PVA consists of two absorption bands peaking at 2.07 $(A)$ and $3.44 \mathrm{eV}(B)[8,9]$. More detailed analysis put forward in $[8,9]$ shows, however, that the spectrum of the solution, as well as that of nanocomposite films obtained from this solution can be better fitted with four Gaussian bands. The positions of the band maxima in the films are shifted towards low energies by $0.01-0.06 \mathrm{eV}$ with respect to the values found for the colloidal solution. The shift is accompanied by the increase (20 to $80 \%$ ) of half-widths of the bands.

A typical absorption spectrum of PbS-PVA nanocomposite films, investigated in this work, is shown in Fig. 2 (curve 1). The low energy $A$ band (close to $2 \mathrm{eV}$ ) is not well pronounced, appearing as a shoulder on the low-energy tail of the more pronounced $B$ band peaking at ca $3.5 \mathrm{eV}$. A weak absorption is also observed down to $1.15 \mathrm{eV}$. A first plausible explanation that could be

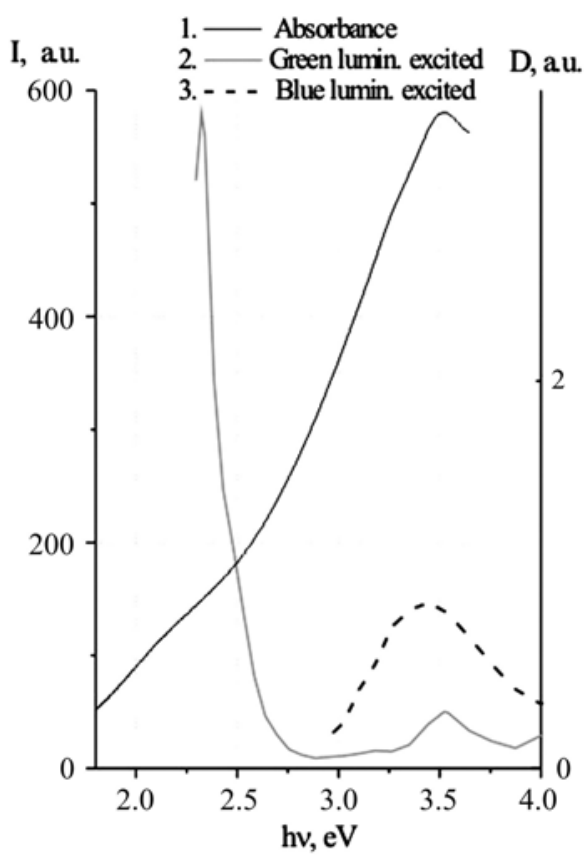

Fig. 2. Absorption spectrum (1) and luminescence excitation spectra of green (2) and blue (3) luminescence in PbS-PVA nanocomposite films. The emission was measured at $2.25 \mathrm{eV}$ for curve 2, and at $3.02 \mathrm{eV}$ for curve 3 . 
offered would assume that the size of the nanocrystallites decreases when drying (e.g., due to removal of water molecules), and simultaneously the intensities of higher energy transitions increase (hence reducing the relative importance of the low energy transition). It would be difficult to explain, however, why the dispersion (manifesting itself in the absorption band halfwidth) increases upon formation of the film, if these nanoparticles have already been formed in the colloidal solution and stabilized by molecules of polymer. Therefore, we suggest that the absorption spectra of our samples would be correctly fitted with four Gaussian bands in the range of strong absorption ( 1.8 to $3.7 \mathrm{eV})$, and by one impurity band in the range of weak absorption (1.0 to $1.8 \mathrm{eV})$. The spectra of PbS-PVA nanocomposite films can be fitted with the bands peaking at $1.5,1.84,2.41,3.06$, and $3.58 \mathrm{eV}$. The literature data for polycrystalline $\mathrm{PbS}$ films [10] report four transitions of various symmetries with maxima around 1.85 to $2.00\left(\mathrm{~L}_{5} \rightarrow \mathrm{L}_{7}\right), 2.20$ to $2.46\left(\Sigma_{5} \rightarrow \Sigma_{6}\right)$, 3.00 to $3.35\left(\Sigma_{5} \rightarrow \Sigma_{7}\right)$ and 3.30 to $3.66 \mathrm{eV}\left(\Delta_{5} \rightarrow \Delta_{6}\right)$. It is evident that the positions of the bands found in our samples coincide with those given in [10], except for the lowest-energy $(1.5 \mathrm{eV})$ band. Thus, the comparison speaks in favour of our hypothesis.

\subsection{Luminescence}

Two kinds of luminescence (hereafter referred to as a green and blue luminescence, respectively) have been observed in the $\mathrm{PbS}$ nanocomposite films under study (cf. Fig. 3).

The green luminescence is dominant in the range of 2.00 to $2.34 \mathrm{eV}$ with the maximum at $2.25 \mathrm{eV}$ and the inflection close to $2.05 \mathrm{eV}$ (Fig. 3, curve 1). In the excitation spectrum of this luminescence, two peaks have been detected with maxima at 2.30 and $3.56 \mathrm{eV}$ (Fig. 2, curve 2). The excitation efficiency of green luminescence with $2.30 \mathrm{eV}$ radiation has been found ca one order of magnitude greater than that with $3.56 \mathrm{eV}$ radiation. Consequently, excitation of green luminescence seems to be associated with $\Delta_{5} \rightarrow \Delta_{6}$ and $\Sigma_{5} \rightarrow \Sigma_{6}$ transitions. The intensity of green luminescence excited with $h v=3.10 \mathrm{eV}$ is low hence the contribution of the $\Sigma_{5} \rightarrow \Sigma_{7}$ transition (3.06 eV) should be small.

The blue luminescence appears in the range 2.20 $3.20 \mathrm{eV}$ with the peak at $3.02 \mathrm{eV}$ and inflections at 2.82 and $2.61 \mathrm{eV}$. The maximum in the excitation spectrum of this luminescence $(3.46 \mathrm{eV})$ is red shifted by $0.1 \mathrm{eV}$ relative to the peak of the $\Delta_{5} \rightarrow \Delta_{6}$ transition (Fig. 3, curve 2). The shift can be caused by a small contribution of the $\Sigma_{5} \rightarrow \Sigma_{7}$ transition $(3.06 \mathrm{eV})$.

A question that should be answered at this point is the one concerning the nature of the centers responsible for the luminescence, one of possible explanations being the presence of impurities or structural defects in the $\mathrm{PbS}$ nanocomposites. The question can be answered by investigating the effect of thermal annealing on the

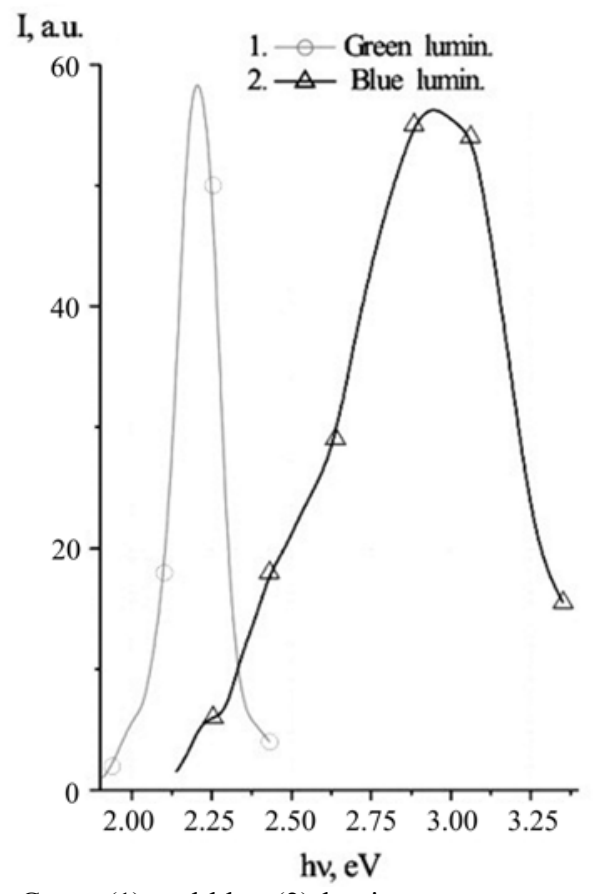

Fig. 3. Green (1) and blue (2) luminescence spectra of PbSPVA nanocomposite films. The excitation wavelengths were equal to 2.30 and $3.46 \mathrm{eV}$ for the curves 1 and 2 , respectively.

spectra; one may expect that the concentrations of impurities (in particular, of water vapors) and defects should decrease with increasing the annealing temperature and/or the prolonging annealing time.

\subsection{Effect of annealing on optical properties}

The annealing experiments reported in this paper were carried out in the air. The annealing time was kept constant (1 hour), whereas we employed several annealing temperatures, $T_{a}$, ranging between 330 and $410 \mathrm{~K}$. The most pronounced changes were observed at $T_{a}=370 \mathrm{~K}$ : the intensity of the green luminescence decreases almost twice after annealing at $370 \mathrm{~K}$, whereas the intensity of the blue luminescence increases. After annealing at various temperatures, the analysis of the absorption spectra shows that the energy of the optical transitions in $\mathrm{PbS}$ nanoparticles remains practically constant, but the relation of the Gaussian component intensities in the absorption spectra noticeably changes. A comparison of the absorption spectra of the films annealed at $T_{a}=330 \mathrm{~K}$ (Fig. 4a) and $410 \mathrm{~K}$ (Fig. 4b) demonstrates that the intensity of the $\Delta_{5} \rightarrow \Delta_{6}$ transition $(3.58 \mathrm{eV})$ increases with increasing $T_{a}$ whereas all the other intensities decrease. These trends are better seen in Fig. 5: the largest relative decrease (ca in half) was observed for the $1.5 \mathrm{eV}$ bands, which we associated with the presence of defects and impurities. This feature seems to confirm that the number of defects and impurity centers decreases in the annealing process. The change of all the other intensities are less pronounced, 
D, a.u.
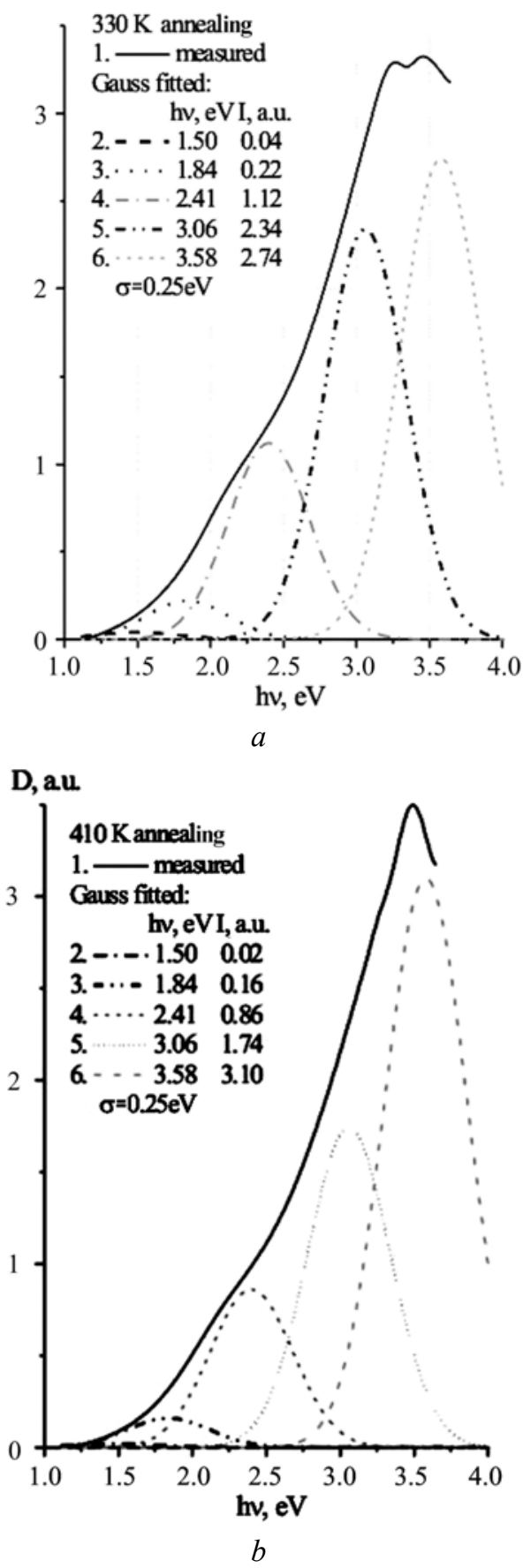

Fig. 4. Absorption spectra of PbS-PVA nanocomposite films and results of their fitting with five Gaussian curves. The samples were annealed in the ambient athmosphere for 1 hour at 330 (a) and $410 \mathrm{~K}(\mathrm{~b})$.

except for $370 \mathrm{~K}$, i.e., for the vicinity of the glass transition temperature of PVA $\left(T_{c}=370 \mathrm{~K}\right)$ [11]. We assume that, at this temperature, removed are residual gases and, above all, water molecules remaining in the polymer film after degassing at $300 \mathrm{~K}$.

Absorption spectra are generally less sensitive to the presence of impurities than the luminescence and excitation ones. In this context, it is necessary to note a correlation between the reduction of the intensity of green luminescence and reduction of the transition intensity at $2.41 \mathrm{eV}$, as well as between the intensity increase of $3.58 \mathrm{eV}$ transition and the increase of blue luminescence intensity. These transitions make basic contributions to the green and blue luminescence excitation, respectively. The correlations allow us to assume that a fraction of the energy absorbed by $\mathrm{PbS}$ nanocrystals is transmitted to local states associated (directly or indirectly) with residual water molecules, resulting in the appearance of the green luminescence.

\subsection{Photovoltaic properties}

The photovoltage $(V)$ of ITO/nano-PbS structures was measured under illumination of the different films sides with the modulated radiation of LEDs. The diodes emitting 1.32, 1.90, 2.18, and $2.64 \mathrm{eV}$ quanta were employed. Upon illumination of the samples through the substrate and the ITO electrode, Processes in the thin layer at the electrode/sample interface $\left(V_{c}\right)$ make the principal contribution to the photovoltage, whereas the illumination of the surface results in the photovoltage caused by the processes occured near the free surface $\left(V_{s}\right)$.

The photovoltaic sensitivity $\eta$ was calculated from the measured photovoltage $(V)$ with the relation

$\eta=\frac{V}{P(1-T)}$,

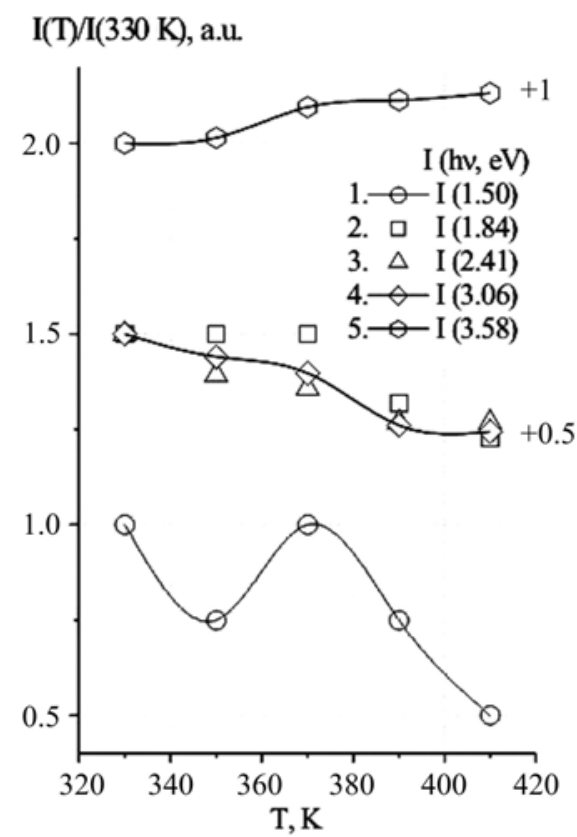

Fig. 5. Dependence of the intensities of the Gaussian bands determined from the deconvolution of the absorption spectra on the annealing temperature. The intensities were normalized to the lowest annealing temperature and vertically shifted; the shifts are indicated on the graph. 

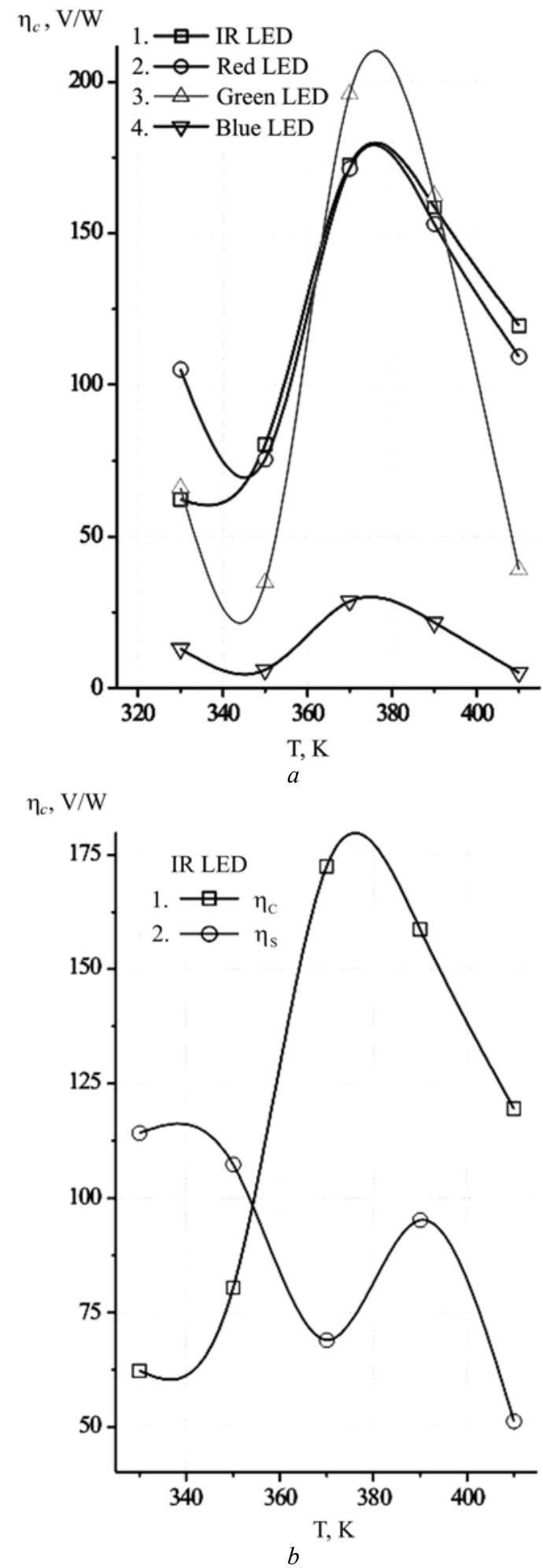

Fig. 6. Dependences of the photovoltaic efficiencies on the annealing temperature of the PbS-PVA nanocomposite. $a$ - The samples were illuminated through the ITO electrode. The curves 1-4 are the dependences measured for the energies of quanta amounted to $1.32,1.90,2.18$ and $2.64 \mathrm{eV}$, respectively.

$b$ - The samples were illuminated with an IR LED $(h v=1.32 \mathrm{eV})$ through the ITO electrode (curve 1) and on the free surface (curve 2). where $P$ is the power of the LED radiation incident onto the sample and $T$ is the transmittance of the sample at the wavelength of the radiation emitted by the LED. We shall refer to the efficiency determined for the samples illuminated through the ITO electrode as $\eta_{c}$, and to the efficiency calculated for the samples whose surface was directly illuminated as $\eta_{s}$. The $\eta_{c}\left(T_{a}\right)$ dependences determined for a few wavelengths are shown in Fig. 6 a. The dependence of the sensitivity on the direction of illumination is shown in Fig. $6 \mathrm{~b}$.

As seen from Fig. $6 \mathrm{a}, \eta_{c}$ depends on the annealing temperature more strongly than on the absorbance (Fig. 5) reaching its maximum for samples annealed at $T_{a}=370 \mathrm{~K}$. It has been also found that $\eta_{s}>\eta_{c}$ in $\mathrm{PbS}$ nanocomposite films before annealing and in samples annealed at low temperatures (Fig. 6b). $\eta_{s}$ decreases as the annealing temperature increases becoming 2 to 3 times lower than $\eta_{c}$ in all the samples annealed at $T_{a}>T_{c}$. It occurs most probably due to the more effective desorption of residual water molecules from the free surface. This conclusion is supported by the presence of the minimum in the curve $\eta_{s}\left(T_{a}\right)$ at $T_{a} \approx T_{c}$ (Fig. 6b), when an effective adsorptions of waters vapors and other gases begins. Hence, energy of absorbed light turn to defects, those generating free charge carriers. Since there are impurities at the surface after annealing when $T_{a}>T_{c}$ becoming less than near the contact (inside the bulk), and $\eta_{s}$ becomes less than $\eta_{c}$.

The obtained data confirm that the photovoltage appears in all polymer-PbS nanocomposite films over a wide spectral range from 1.3 up to $2.7 \mathrm{eV}$. Consequently, the spectral range of photosensitivity of the studied $\mathrm{PbS}$ nanocomposite films is wider than that of polymer-CdSe and $\mathrm{CuInS}_{2}$ nanocomposite films [4].

\section{Conclusions}

The absorption spectra of $\mathrm{PbS}$ nanocomposite films measured in the range of 1.0 to $3.7 \mathrm{eV}$ are correctly fitted with five Gaussian bands peaking 1.5, 1.84, 2.41, 3.06 , and $3.58 \mathrm{eV}$. Annealing in the air weakly changes relative intensities of the bands but practically does not affect their energy.

The basic feature of $\mathrm{PbS}$ nanocomposite films is the presence of green and blue luminescence with intensity maxima at 2.25 and $3.02 \mathrm{eV}$, respectively. The luminescence intensity strongly depends on the energy of excitation and on the annealing temperature. The maximum intensity of the green luminescence is observed upon excitation with $2.30 \mathrm{eV}$ radiation, and that of the blue emission - upon excitation with $3.46 \mathrm{eV}$ quanta. Thus, the green luminescence can be associated with the electronic transition at $2.41 \mathrm{eV}$, and the blue one - with $3.58 \mathrm{eV}$. After annealing at glass transition temperature $(370 \mathrm{~K})$, the intensity of the green luminescence is few times decreased, whereas the blue emission intensity increases. 
$\mathrm{PbS}$ nanocomposite films have been found to exhibit the photovoltaic activity over the spectral range from 1.3 to $3.7 \mathrm{eV}$ at least. Their photovoltaic sensitivity was found to reach a maximum after annealing in air at $370 \mathrm{~K}$, i.e., at about the PVA glass transition temperature. The spectral range of photosensitivity of $\mathrm{PbS}$ nanocomposite films under study is wider than that of polymer-CdSe or $\mathrm{CuInS}_{2}$ nanocomposite films.

\section{References}

1. R.K. Jain, R.C. Lind, Degenerate four-wave mixing in semiconductor-doped glasses // J. Opt. Soc. Amer. 73, p. 646-650 (1983).

2. T. Fukumi, T. Sakaguchi, S. Yanagida, T. Enokida, Nonlinear Optical Materials. Boca Raton, CRC Press, London (1992).

3. C.J. Brabec, N.C. Sariciftci, J.C. Hummelen, Plastic solar cells // Adv. Funct. Mater. 11, p. 15-26 (2001).

4. E. Arici, N.S. Sariciftci, D. Meissner, Photovoltaic properties of nanocrystalline $\mathrm{CuInS}_{2} /$ methanofullerene solar cells // Mol. Cryst. Liquid Cryst. 385, p. 129-136 (2002).
5. A.S. Kutsenko, S.M. Maloletov, S.Ya. Kuchmii, V.R. Lyakhovetskii, and V.I. Volkov, Third-order nonlinear optical response of a new PVA composite with $\mathrm{PbS}$ nanocrystals // Theor. Exper. Chem. 38, p. 173-178 (2002).

6. H.P. Klug, L.E. Alexander, X-ray diffraction procedures. John Wiley, New York (1954).

7. I.A. Akimov, Study of internal photoeffect in semiconductors by using capacitor method // Optikomechanicheskaya Promyshlennost' 5, p. 4-13 (1966) (in Russian).

8. J-J. Legendre, J. Livage, Vanadium pentoxide gels: I. Structural study by electron diffraction // J. Colloid Interface Sci. 94, p. 73-83 (1983).

9. S. Gallardo, M. Gutierrez, A. Henglein, E. Janata, Photochemistry and radiation chemistry of colloidal semiconductors. Properties of Q-PbS // Ber. Bunsenges. Phys. Chem. 93, p. 1080-1090 (1989).

10. V.I. Gavrilenko, A.M. Grechov, D.V. Korbutyak, V.T. Lytovchenko, Optical properties of semiconductors. Naukova Dumka, Kyiv (1987) (in Russian).

11. S.N. Ushakov, Polyvinyl alcohol and its derivatives, Acad. Sci. USSR Publ., Moscow-Leningrad (1960). 